\title{
Photosynthesis rate, salinity induced grazing rate and gestation delay as determinants of the dynamics of a realistic food chain model: a stochastic approach
}

\author{
Ujjwal Roy $^{1}$ N. C. Majee ${ }^{1} \cdot$ Santanu Ray ${ }^{2}$
}

Received: 8 February 2016/Accepted: 18 March 2016/Published online: 2 April 2016

(C) Springer International Publishing Switzerland 2016

\begin{abstract}
Impact of photosynthesis rate of phytoplankton, salinity induced grazing rate of zooplankton and gestation delay of zooplankton and fish population are considered in the present study. Models with delay are much more realistic, as in reality time delays occur in almost every biological situation and assume to be one of the reasons of regular fluctuations in population density. Reproduction of predator after consuming prey is not instantaneous, but mediated by some time lag required for gestation. It is observed that there is stability switches and Hopf bifurcation occurs when the delay crosses some critical value. It is observed that the quantitative level of abundance of system population depends crucially on the delay parameter if the gestation period exceeds some critical value. Also, environmental stochasticity in the form of Gaussian whitenoise, plays a significant role to describe the system and its values. Numerical examples are also support the model assumption and analytical results.
\end{abstract}

Keywords Photosynthesis rate $\cdot$ Salinity induced grazing rate $\cdot$ Gestation delay $\cdot$ Hopf-bifurcation - Gaussian white-noise

Ujjwal Roy

ujjwal2ju@gmail.com

1 Department of Mathematics, Jadavpur University, Kolkata 700 032, India

2 Ecological Modelling Laboratory, Department of Zoology, Visva Bharati University, Santiniketan 731 235, India

\section{Introduction}

Ecosystem is composed of dynamically interacting parts including organisms, the communities they make up and the non-living components of their environment. Understanding the dynamical relationship between prey and predator through mathematical modelling in theoretical ecology has gained a lot of importance during the last few decades (Berryman 1992). On the purpose of observation and study the effect of environmental noise on population dynamics, researchers have used Gaussian white-noise (Lande 1993; May 1973; Jana et al. 2015) and color noise (Samanta and Maiti 2003; Jana and Bairagi 2013) as a model of environmental variations. May (1973) studies a population model considering white noise and shows that when the population deviates more from equilibrium point, the system shows irregular behavior (i.e., instability). Where as Jana et al. (2015) show a three species food chain model where the interacting processes among the species are influenced by Gaussian white-noise and with increasing noise intensity system gradually goes into chaos from stable coexistence through instability stochastically. Ripa et al. (1998) examine the effect of environmental noise on population and present a general theory of environmental noise in ecological food webs. On a fairly realistic ecological model with generalist top predator, Upadhyay et al. (2007) investigate the influence of environmental noise and show the importance of the noise amplitude on the trophic level and the susceptibility of populations to environmental noise. Complex dynamical behavior of a biological system arises as a consequence of time delay (with significant time delay) which may exhibit limit cycle oscillations (Ruan 2009) and chaos (Mackey and Glass 1977; Jana 2014; Jana et al. 2014). In reality, time delays occur in almost every ecological situation and assume to be one of the most 
prominent reason of regular fluctuations on population biomass (Jana 2014; Jana et al. 2014, 2015). Ruan (2009) presents a brief review on prey-predator models with descrete delay for both Kolmogorov and non-Kolmogorov type model system. It often represents gestation time (Jana 2014; Jana et al. 2014), incubation period, transport delay or can simply lump complicated biological process together, accounting only for the time requires for these process to occur. Delay parameter emphasizes on Hopf-bifurcation (Ruan 2009; Jana 2014; Jana et al. 2014, 2015) and also it causes some complex dynamical behaviors, such as double Hopf bifurcation, quasi-periodic solutions and chaos (Lü 2002; Ma et al. 2008; Matouk 2008; Ding and Jiang 2011; Wang et al. 2014; Ding et al. 2013; Song and Xu 2013). Along with different studies of environmental stochasticity and biological delays in prey-predator system, influence of noise on a delayed system is very significant for advance study of biological system. Schwartz et al. (2015) and Jana et al. (2015) study the effect of delay on the rates of noiseinduced switching between co-existing stable states and noise-induced extinction in a population dynamic model.

In the present work, we have investigated the complex dynamical behavior of a phytoplankton-zooplankton-fish population delayed food chain model with Holling type II functional response as to describe feeding process of both zooplankton and fish population. Here we consider gestation delays in the numerical response terms of both zooplankton and fish population. The paper is structured as follows: in "Model and analysis", model system and their stability and Hopf-bifurcation has been discussed, followed by "Stochastic scenario of non-delayed system", "Stochastic scenario of delayed system" and "Numerical results". At last, in "Discussion", conclusions and discussions have been made.

\section{Model and analysis}

Let $P, Z$ and $F$ denote the population of phytoplankton, zooplankton and fish population respectively. The model is

$$
\begin{aligned}
& \frac{d P}{d t}=m_{1} P\left(1-\frac{P}{K_{P}}\right)-g_{s} \frac{P Z}{P+K_{Z}}, \\
& \frac{d Z}{d t}=a g_{s} \frac{P Z}{P+K_{Z}}-m_{2} Z-g_{w} \frac{Z F}{Z+K_{F}}, \\
& \frac{d F}{d t}=g_{w} \frac{Z F}{Z+K_{F}}-m_{3} F .
\end{aligned}
$$

In this present $P Z F$ model, light and temperature dependant photosynthesis rate $\left(m_{1}\right)$ of phytoplankton and salinity induced grazing rate $\left(g_{s}\right)$ of zooplankton are incorporated, these equations are taken from the model of Mandal et al. (2012). $m_{1}=\left(r_{p h}+r_{s}+M_{p h}\right)$,

where, $r_{p h}, r_{s}, M_{p h}$ are respectively respiratory rate of phytoplankton, rate of settling and mortality rate of phytoplankton.

$g_{s}=\delta g_{z}$

where

$$
\delta=\frac{s_{u}}{s_{u}-s_{d}},
$$

where, $g_{s}, S_{d}, S_{u}, g_{z}, g_{w}, K_{Z}, K_{F}$ and $K_{P}$ are salinity induced grazing of zooplankton, upstream salinity, downstream salinity, grazing rate of zooplankton, grazing rate of fish, half saturation constant of zooplankton on phytoplankton, half saturation constant of fish on zooplankton and environmental carrying capacity of phytoplankton. Since estuary is a transition zone of river and sea, there is always fluctuation of salinity throughout the year, which is due to dilution by upstream river water and/or mixing by downstream sea tidal water. $\delta$ is the dilution factor, is calculated following the equation of Ketchum (1951). It is the function of $S_{d}$ and $S_{u}$. Besides grazing the abundance of zooplankton is also dependent on loss due to $E_{z o o}=Z E_{z o}$, respiration $R_{z o o}=Z r_{z o}$, fish predation $F_{p}=Z r_{r f}$, and mortality $M_{z o o}=Z M_{z}, R_{z o o}$ is governed by $r_{z o}$.

$m_{2}=\left(E_{z o}+r_{z o}+r_{f p}+M_{z}\right)$.

The abundance of $F$ is governed by many processes in the estuary. Fish predation $F_{p}$ on $Z$ follows Michaelis-Menten kinetics (type II) which enriches the fish pool of the estuarine system. $F_{p}$ depends on $K_{Z}$ and $g_{f} . F$ population is reduced by mortality rate of fish $M_{f}$, respiration $F r_{f}$, harvest by fishing $F H_{c f}$ and excretion of fish $F E_{f}$ and $F H_{f}$ are controlled by $R_{f}$ and $H_{f}$ respectively. Respiration rate of fish and $H_{f}$ harvest rate of carnivorous fish respectively.

$$
m_{3}=\left(E_{f}+M_{f}+r_{f}+H_{f}\right) .
$$

It is already mentioned that time-delay is an important factor in biological systems. As a starting point of this section, we consider the following generalization of the model

$$
\begin{aligned}
& \frac{d P}{d t}=m_{1} P\left(1-\frac{P}{K_{P}}\right)-g_{s} \frac{P Z}{P+K_{Z}}, \\
& \frac{d Z}{d t}=a g_{s} \frac{P(t-\tau) Z}{P(t-\tau)+K_{Z}}-m_{2} Z-g_{w} \frac{Z F}{Z+K_{F}}, \\
& \frac{d F}{d t}=g_{w} \frac{Z(t-\tau) F}{Z(t-\tau)+K_{F}}-m_{3} F,
\end{aligned}
$$

where $\tau$ represents the gestation period of zooplankton and fish population respectively. 
The system (7) has only one interior equilibrium point given by $E_{*}=\left(P^{*}, Z^{*}, F^{*}\right)$; where

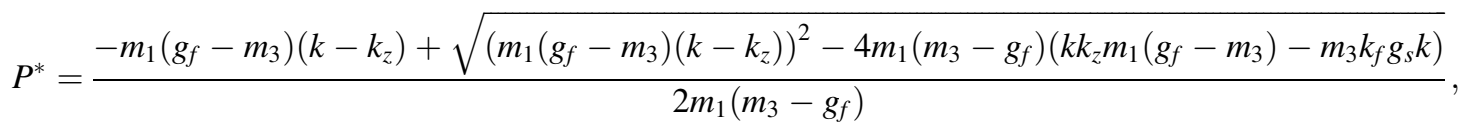

$Z^{*}=\frac{m_{3} k_{f}}{\left(g_{f}-m_{3}\right)}$ and $F^{*}=\frac{m_{1} k_{f} a\left(P^{*}-k\right)}{m_{3} k}+\frac{k_{f}\left(a g_{s}-m_{2}\right)}{\left(g_{f}-m_{3}\right)}$. The equilibrium point $E^{*}$ is biologically feasible if $\left(g_{f}-m_{3}\right)>0$. Linearizing the system (7) at $E^{*}$, we get

$\frac{d P}{d t}=a_{11} P(t)+a_{12} Z(t)$

$\frac{d Z}{d t}=a_{22} Z(t)+a_{23} F(t)+b_{21} P(t-\tau)$,

$\frac{d F}{d t}=b_{32} Z(t-\tau)$

where, $\quad a_{11}=-m_{1} \frac{P^{*}}{K_{P}}+g_{s} \frac{P^{*} Z^{*}}{\left(P^{*}+K_{Z}\right)^{2}}, a_{12}=g_{s} \frac{P^{*}}{P^{*}+K_{Z}}, a_{22}=$ $g_{w} \frac{Z^{*} F^{*}}{\left(Z^{*}+K_{F}\right)^{2}}, \quad a_{23}=g_{w} \frac{Z^{*}}{Z^{*}+K_{F}}, b_{21}=a g_{s} \frac{K_{Z} Z^{*}}{\left(Z^{*}+K_{Z}\right)^{2}}, b_{32}=g_{w}$ $\frac{F^{*} K_{F}}{\left(Z^{*}+K_{F}\right)^{2}}$. The corresponding characteristic equation is given by

$\lambda^{3}+A \lambda^{2}+B \lambda+e^{-\lambda \tau}(C \lambda+D)=0$,

where $A=-\left(a_{11}+a_{22}\right), B=a_{11} a_{22}, C=-\left(a_{23} b_{32}+a_{12}\right.$ $\left.b_{21}\right)$ and $D=a_{11} a_{23} b_{32}$. We now discuss the following cases.

Case I: $\tau=0$

For non-delayed system i.e., when $\tau=0$, characteristic Eq. (9) becomes

$\lambda^{3}+A \lambda^{2}+(B+C) \lambda+D=0$.

Hence, by Routh-Hurwitz criterion, we get the stability conditions of $E^{*}$.

Theorem 1 The coexistence equilibrium point $E^{*}$ of system (1) is asymptotically stable if $A, B+C, D>0$ and $A(B+C)>D$.

Case II: $\tau>0$

We know that $i \omega(\omega>0)$ is a root of (9) if and only if $\omega$ satisfies

$-i \omega^{3}-A \omega^{2}+B i \omega+(i C \omega+D)(\cos \omega \tau-i \sin \omega \tau)=0$.

Separating real and imaginary parts, we get
$A \omega^{2}=C \omega \sin \omega \tau+D \cos \omega \tau$,

$S \omega^{3}+B \omega=D \sin \omega \tau-C \omega \cos \omega \tau$.

These two equations give the positive values of $\tau$ and $\omega$ for which Eq. (9) can have purely imaginary roots. Squaring and adding these equations, we have

$\omega^{6}+p \omega^{4}+q \omega^{2}+r=0$,

where

$p=A^{2}+2 B$,

$q=B^{2}-C^{2}$,

$r=-D^{2}$.

Now we shall measure the value of $\tau$ for which system (7) will remain stable. From Eq. (11) we have,

$\tau_{k}=\frac{1}{\omega_{k}}\left[\cos ^{-1}\left\{\frac{(A D-B C) \omega_{k}^{2}-C \omega_{k}^{4}}{D^{2}+C^{2} \omega_{k}^{2}}\right\}+2 k \pi\right]$,

$k=0,1,2,3, \ldots$, where $\omega_{k}$ is the real positive root of Eq. (12). Also, differentiating Eq. (9) with respect to $\tau$ we get

$\left[\operatorname{Re}\left(\frac{d \lambda}{d \tau}\right)^{-1}\right]_{\tau=\tau_{0}}=\frac{U_{1} V_{1}+U_{2} V_{2}}{V_{1}^{2}+V_{2}^{2}}$

where,

$$
\begin{aligned}
U_{1}= & C+\sin \omega \tau\left(-2 A \omega+\tau \omega^{3}-B \tau \omega\right) \\
& +\cos \omega \tau\left(B-3 \omega^{2}-A \tau \omega^{2}\right), \\
U_{2}= & \sin \omega \tau\left(B-3 \omega^{2}-A \tau \omega^{2}\right) \\
& +\cos \omega \tau\left(2 A \omega-\tau \omega^{3}+B \tau \omega\right), \\
V_{1}= & -A \omega^{3} \sin \omega \tau+\cos \omega \tau\left(B \omega^{2}-\omega^{4}\right), \\
V_{2}= & \sin \omega \tau\left(B \omega^{2}-\omega^{4}\right)+A \omega^{3} \cos \omega \tau .
\end{aligned}
$$

Hence the transversality condition is satisfied if $\left(U_{1} V_{1}+U_{2} V_{2}\right)>0$.

Theorem 2 Assume $\tau>0$ and conditions of the Theorem 1 are satisfied, then the equilibrium $E^{*}\left(P^{*}, Z^{*}, F^{*}\right)$ is locally asymptotically stable for $\tau<\tau_{0}$ and unstable for $\tau>\tau_{0}$. Furthermore, the system (7) undergoes a Hopf bifurcation at $E^{*}\left(P^{*}, Z^{*}, F^{*}\right)$ when $\tau=\tau_{0}$, where 
$\tau_{0}=\frac{1}{\omega_{0}}\left[\cos ^{-1}\left\{\frac{(A D-B C) \omega_{0}^{2}-C \omega_{0}^{4}}{D^{2}+C^{2} \omega_{0}^{2}}\right\}\right]$.

\section{Stochastic scenario of non-delayed system}

Deterministic models in ecology do not usually incorporate environmental fluctuation; they are often justified by the implicit assumption that in large population, stochastic deviations are small enough to be ignored. Deterministic model will prove ecologically useful only if the dynamical patterns they reveal are still in evidence when stochastic effects are introduced. For terrestrial system, the environmental variability is large at both short and long time periods and could be expected to develop internal mechanisms to the system which would cope with short term variability and minimize the effects of long term variations, hence analysis of the system with white noise gives better results. Uncertain growth of populations is usually considered as an effect of environmental stochasticity. Reproduction of species depends on various factors, such as temperature, humidity, parasites and pathogens, environmental pollution etc. (Iwasa et al. 2000). Since physical and biological environments of populations are not totally predictable, the growth of populations should be considered as a stochastic process rather than a deterministic one (Turelli 1986). In spite of some shortcomings, Gaussian white noise has been proved extremely useful to model rapidly fluctuating phenomena (Valsakumar et al. 1983; Samanta and Maiti 2003; Jana et al. 2015). Therefore, in the non-delayed counterpart of model system (1) by introducing the environmental stochasticity in the form of Gaussian white noise is represented by:

$$
\begin{aligned}
& \frac{d P}{d t}=m_{1} P\left(1-\frac{P}{K_{P}}\right)-g_{s} \frac{P Z}{P+K_{Z}}+\alpha_{1} \eta_{1}(t), \\
& \frac{d Z}{d t}=a g_{s} \frac{P Z}{P+K_{Z}}-m_{2} Z-g_{w} \frac{Z F}{Z+K_{F}}+\alpha_{2} \eta_{2}(t), \\
& \frac{d F}{d t}=g_{w} \frac{Z F}{Z+K_{F}}-m_{3} F+\alpha_{3} \eta_{3}(t),
\end{aligned}
$$

where the perturbed terms $\eta_{1}(t), \eta_{2}(t)$ and $\eta_{3}(t)$ are assumed to be the independent Gaussian white noise satisfying the conditions:

$$
\left\langle\eta_{j}(t)\right\rangle=0 \quad \text { and } \quad\left\langle\eta_{j}\left(t_{1}\right) \eta_{j}\left(t_{2}\right)\right\rangle=\epsilon_{i j} \delta\left(t_{1}-t_{2}\right)
$$

for $j=1,2,3$.

Here $\epsilon_{i j}>0(j=1,2,3)$ are the intensities or strengths of the random perturbations (Kronecker symbol), $\delta$ is the Dirac delta function defined by

$\left\{\begin{array}{l}\delta(x)=0, \\ \int_{-\infty}^{\infty} \delta(x) d x=1,\end{array} \quad\right.$ for $\mathrm{x} \neq 0$ $\langle\cdot\rangle$ represents the ensemble average of the stochastic process and $\alpha_{i}(i=1,2,3)$ are real constants. It is noted that $\eta_{i}(t)$ are not defined in the ordinary sense. It can be proved that $\eta_{i}(t)$ are the derivatives of the Wiener process $W_{i}(t)$ in the generalized functions sense. To study the behavior of the system (17) about the steady state $E^{*}$, let us substitute $P^{\prime}=\ln P, \quad Z^{\prime}=\ln Z, \quad F^{\prime}=\ln F ; \quad P=u+P^{*} Z=$ $v+Z^{*}$ and $\mathrm{F}=\mathrm{w}+\mathrm{F}^{*}$. Then the system (17) reduces to the following linear stochastic differential equations in terms of deviation variables $(u, v, w)$ :

$$
\begin{aligned}
& \frac{d u}{d t}=\alpha_{11} P^{*} u+\alpha_{12} P^{*} v+\alpha_{13} P^{*} w+\alpha_{1} \eta_{1}(t), \\
& \frac{d v}{d t}=\alpha_{21} Z^{*} u+\alpha_{22} Z^{*} v+\alpha_{23} Z^{*} w+\alpha_{2} \eta_{2}(t), \\
& \frac{d w}{d t}=\alpha_{31} F^{*} u+\alpha_{32} F^{*} v+\alpha_{33} F^{*} w+\alpha_{3} \eta_{3}(t),
\end{aligned}
$$

where

$$
\begin{aligned}
& \alpha_{11}=-m_{1} \frac{P^{*}}{K_{P}}+g_{s} \frac{P^{*} Z^{*}}{\left(P^{*}+K_{Z}\right)^{2}}, \quad \alpha_{12}=g_{s} \frac{1}{P^{*}+K_{Z}}, \quad \alpha_{13}=0, \\
& \alpha_{21}=a g_{s} \frac{K_{Z}}{\left(Z^{*}+K_{Z}\right)^{2}}, \quad \alpha_{22}=g_{w} \frac{F^{*}}{\left(Z^{*}+K_{F}\right)^{2}}, \quad \alpha_{23}=g_{w} \frac{1}{Z^{*}+K_{F}}, \\
& \alpha_{31}=0, \quad \alpha_{32}=g_{w} \frac{K_{F}}{\left(Z^{*}+K_{F}\right)^{2}}, \quad \alpha_{33}=0 .
\end{aligned}
$$

The solutions $(u(t), v(t), w(t))$ of system (18) subject to known initial values $\left(u\left(t_{0}\right), v\left(t_{0}\right), w\left(t_{0}\right)\right)$ determine the statistical behavior of the model system (17) near the steady state $E^{*}$ at time $t>t_{0}$.

Taking the Fourier transform on both sides of (18), we get,

$$
\begin{aligned}
& \alpha_{1} \tilde{\eta}_{1}(\omega)=\left(i \omega+\alpha_{11} P^{*}\right) \tilde{u}(\omega)+\alpha_{12} P^{*} \tilde{v}(\omega)+\alpha_{13} P^{*} \tilde{w}(\omega), \\
& \alpha_{2} \tilde{\eta}_{2}(\omega)=\alpha_{21} Z^{*} \tilde{u}(\omega)+\left(i \omega+\alpha_{22} Z^{*}\right) \tilde{v}(\omega)+\alpha_{23} Z^{*} \tilde{w}(\omega), \\
& \alpha_{3} \tilde{\eta}_{3}(\omega)=\alpha_{31} F^{*} \tilde{u}(\omega)+\alpha_{32} F^{*} \tilde{v}(\omega)+\left(i \omega+\alpha_{33} F^{*}\right) \tilde{w}(\omega) .
\end{aligned}
$$

The matrix of (20) is

$$
M(\omega) \tilde{U}(\omega)=\tilde{\eta}(\omega),
$$

where

$$
\begin{aligned}
& M(\omega)=\left(\begin{array}{ccc}
i \omega+\alpha_{11} P^{*} & \alpha_{12} P^{*} & \alpha_{13} P^{*} \\
\alpha_{21} Z^{*} & i \omega+\alpha_{22} Z^{*} & \alpha_{23} Z^{*} \\
\alpha_{31} F^{*} & \alpha_{32} F^{*} & i \omega+\alpha_{33} F^{*}
\end{array}\right), \\
& \tilde{U}(\omega)=\left(\begin{array}{c}
\tilde{u}(\omega) \\
\tilde{v}(\omega) \\
\tilde{w}(\omega)
\end{array}\right), \tilde{\eta}(\omega)=\left(\begin{array}{c}
\tilde{\eta}_{1}(\omega) \\
\tilde{\eta}_{2}(\omega) \\
\tilde{\eta}_{3}(\omega)
\end{array}\right) .
\end{aligned}
$$

Equation (21) can also be written as $\tilde{U}(\omega)=$ $[M(\omega)]^{-1} \tilde{\eta}(\omega)$. Let $[M(\omega)]^{-1}=K(\omega)$, therefore 


$$
\tilde{U}(\omega)=K(\omega) \tilde{\eta}(\omega)
$$

where

$$
K(\omega)=\frac{\operatorname{Adj}(\mathrm{M}(\omega))}{|M(\omega)|} .
$$

If the function $Y(t)$ has a zero mean value, then the fluctuation intensity (variance) of it in the frequency interval $[\omega, \omega+d \omega]$ is $S_{Y}(\omega) d \omega$. Where $S_{Y}(\omega)$ is spectral density of $Y$ and is defined as

$S_{Y}(\omega)=\lim _{\tilde{T} \rightarrow \infty} \frac{|\tilde{Y}(\omega)|^{2}}{\tilde{T}}$.

The power spectrum of a time series describes how the variance of the data is distributed over the frequency components into which the data may be decomposed. Intuitively, the spectrum decomposes the content of a signal or of a stochastic process into the different frequencies present in that process and helps to identify periodicities. If $Y$ has a zero mean value, the inverse transform of $S_{Y}(\omega)$ is the auto covariance function

$$
C_{Y}(\tau)=\frac{1}{2 \pi} \int_{-\infty}^{\infty} S_{Y}(\omega) e^{i \omega \tau} d \omega
$$

The corresponding variance of fluctuations in $Y(t)$ is given by

$$
\sigma_{Y}^{2}=C_{Y}(0)=\frac{1}{2 \pi} \int_{-\infty}^{\infty} S_{Y}(\omega) d \omega
$$

and the auto correlation function is the normalized auto covariance

$$
P_{Y}(\tau)=\frac{C_{Y}(\tau)}{C_{Y}(0)}
$$

For a Gaussian white noise process, it is

$$
\begin{aligned}
S_{\eta_{i} \eta_{j}}(\omega) & =\lim _{\hat{T} \rightarrow+\infty} \frac{\left\langle\tilde{\eta}_{i}(\omega) \tilde{\eta}_{j}(\omega)\right\rangle}{\hat{T}} \\
& =\lim _{\hat{T} \rightarrow+\infty} \frac{1}{\hat{T}} \int_{-\frac{\hat{T}}{2}}^{\frac{\hat{T}}{2}} \int_{-\frac{\hat{T}}{2}}^{\frac{\hat{T}}{2}}\left\langle\tilde{\eta}_{i}(t) \tilde{\eta}_{j}\left(t^{\prime}\right)\right\rangle e^{-i \omega\left(t-t^{\prime}\right)} d t d t^{\prime}=\delta_{i j} .
\end{aligned}
$$

From (22), we have

$$
\tilde{U}(\omega)=\sum_{j=1}^{2} K_{i j}(\omega) \tilde{\eta}_{j}(\omega), \quad i=1,2,3 .
$$

And from (24), we have

$$
S_{U}(\omega)=\sum_{j=1}^{2} \xi_{j}\left|K_{i j}(\omega)\right|^{2}, \quad i=1,2,3 .
$$

Intensities of fluctuations or variance measures how far a set of numbers is spread out. A variance of zero indicates that all the values are identical. A small variance indicates that the data points tend to be very close to the mean (expected value, here the equilibrium density of prey and predator population) and hence to each other, while a high variance indicates that the data points are very spread out around the mean and from each other. Hence by (26) and (30), the intensities of fluctuations in the variable $U$ are given by

$\sigma_{U}^{2}=\frac{1}{2 \pi} \sum_{j=1}^{2} \int_{-\infty}^{\infty} \alpha_{j}\left|K_{i j}(\omega)\right|^{2} d \omega, \quad i=1,2,3$.

and by (23), we obtain

$$
\begin{aligned}
& \sigma_{u}^{2}=\frac{1}{2 \pi}\left\{\int_{-\infty}^{\infty} \alpha_{1}\left|\frac{A_{1}}{|M(\omega)|}\right|^{2} d \omega+\int_{-\infty}^{\infty} \alpha_{2}\left|\frac{B_{1}}{|M(\omega)|}\right|^{2} d \omega+\int_{-\infty}^{\infty} \alpha_{3}\left|\frac{C_{1}}{|M(\omega)|}\right|^{2} d \omega\right\}, \\
& \sigma_{v}^{2}=\frac{1}{2 \pi}\left\{\int_{-\infty}^{\infty} \alpha_{1}\left|\frac{A_{2}}{|M(\omega)|}\right|^{2} d \omega+\int_{-\infty}^{\infty} \alpha_{2}\left|\frac{B_{2}}{|M(\omega)|}\right|^{2} d \omega+\int_{-\infty}^{\infty} \alpha_{3}\left|\frac{C_{2}}{|M(\omega)|}\right|^{2} d \omega\right\}, \\
& \sigma_{w}^{2}=\frac{1}{2 \pi}\left\{\int_{-\infty}^{\infty} \alpha_{1}\left|\frac{A_{3}}{|M(\omega)|}\right|^{2} d \omega+\int_{-\infty}^{\infty} \alpha_{2}\left|\frac{B_{3}}{|M(\omega)|}\right|^{2} d \omega+\int_{-\infty}^{\infty} \alpha_{3}\left|\frac{C_{3}}{|M(\omega)|}\right|^{2} d \omega\right\}
\end{aligned}
$$

where $|M(\omega)|=|R(\omega)|+i|l(\omega)|$ and real part of $|M(\omega)|$ is

$$
\begin{aligned}
R^{2}(\omega)= & -\alpha_{33} \omega^{2} F^{*}-\alpha_{22} \omega^{2} Z^{*}-\alpha_{11} P^{*}+\left(\alpha_{11} \alpha_{22} \alpha_{33}\right. \\
& +\alpha_{11} \alpha_{32} \alpha_{23}-\alpha_{12} \alpha_{21} \alpha_{33}-\alpha_{12} \alpha_{31} \alpha_{23} \\
& \left.+\alpha_{13} \alpha_{21} \alpha_{32}+\alpha_{13} \alpha_{31} \alpha_{22}\right) P^{*} Z^{*} F^{*} .
\end{aligned}
$$

Imaginary part of $|M(\omega)|$ is

$$
\begin{aligned}
l^{2}(\omega)= & -\omega^{3}+\omega\left(\alpha_{22} \alpha_{33}+\alpha_{32} \alpha_{23}\right) Z^{*} F^{*} \\
& +\omega\left(\alpha_{11} \alpha_{33}+\alpha_{13} \alpha_{31}\right) P^{*} F^{*}+\omega\left(\alpha_{11} \alpha_{22}-\alpha_{12} \alpha_{21}\right) P^{*} Z^{*},
\end{aligned}
$$

and

$$
\begin{array}{lll}
\left|A_{1}\right|^{2}=X_{1}^{2}+Y_{1}^{2}, & \left|B_{1}\right|^{2}=X_{2}^{2}+Y_{2}^{2}, & \left|C_{1}\right|^{2}=X_{3}^{2}+Y_{3}^{2}, \\
\left|A_{2}\right|^{2}=X_{4}^{2}+Y_{4}^{2}, & \left|B_{2}\right|^{2}=X_{5}^{2}+Y_{5}^{2}, & \left|C_{2}\right|^{2}=X_{6}^{2}+Y_{6}^{2}, \\
\left|A_{3}\right|^{2}=X_{7}^{2}+Y_{7}^{2}, & \left|B_{3}\right|^{2}=X_{8}^{2}+Y_{8}^{2}, & \left|C_{3}\right|^{2}=X_{9}^{2}+Y_{9}^{2},
\end{array}
$$

where,

$$
\begin{aligned}
& X_{1}^{2}=-\omega^{2}+\alpha_{32} \alpha_{23} Z^{*} F^{*}, Y_{1}^{2}=\omega \alpha_{33} F^{*}+\omega \alpha_{22} Z^{*}, \\
& X_{2}^{2}=-\left(\alpha_{12} \alpha_{33}+\alpha_{13} \alpha_{32}\right) P^{*} F^{*}, Y_{2}^{2}=-\omega \alpha_{12} P^{*}, \\
& X_{3}^{2}=\left(\alpha_{12} \alpha_{23}-\alpha_{13} \alpha_{22}\right) P^{*} Z^{*}, Y_{3}^{2}=-\omega \alpha_{13} P^{*}, \\
& X_{4}^{2}=-\left(\alpha_{21} \alpha_{33}+\alpha_{23} \alpha_{31}\right) Z^{*} F^{*}, Y_{4}^{2}=-\omega \alpha_{21} Z^{*}, \\
& X_{5}^{2}=-\omega^{2}+\left(\alpha_{11} \alpha_{33}+\alpha_{31} \alpha_{13}\right) P^{*} F^{*}, Y_{5}^{2}=\omega\left(\alpha_{33} F^{*}+\alpha_{11} P^{*}\right), \\
& X_{6}^{2}=\left(\alpha_{21} \alpha_{13}-\alpha_{11} \alpha_{23}\right) P^{*} Z^{*}, Y_{6}^{2}=-\omega \alpha_{23} Z^{*}, \\
& X_{7}^{2}=\left(\alpha_{31} \alpha_{22}-\alpha_{21} \alpha_{32}\right) Z^{*} F^{*}, Y_{7}^{2}=\omega \alpha_{31} F^{*}, \\
& X_{8}^{2}=\left(\alpha_{11} \alpha_{32}-\alpha_{12} \alpha_{31}\right) P^{*} F^{*}, Y_{8}^{2}=\omega \alpha_{32} F^{*}, \\
& X_{9}^{2}=-\omega^{2}+\left(\alpha_{11} \alpha_{22}-\alpha_{12} \alpha_{21}\right) P^{*} Z^{*}, Y_{9}^{2}=\omega\left(\alpha_{22} Z^{*}+\alpha_{11} P^{*}\right) .
\end{aligned}
$$


Thus (32) becomes

$$
\begin{aligned}
\sigma_{u}^{2}= & \frac{1}{2 \pi}\left\{\int _ { - \infty } ^ { \infty } \frac { 1 } { R ^ { 2 } ( \omega ) + l ^ { 2 } ( \omega ) } \left[\alpha_{1}\left(X_{1}^{2}+Y_{1}^{2}\right)\right.\right. \\
& \left.\left.+\alpha_{2}\left(X_{2}^{2}+Y_{2}^{2}\right)+\alpha_{3}\left(X_{3}^{2}+Y_{3}^{2}\right)\right] d \omega\right\}, \\
\sigma_{v}^{2}= & \frac{1}{2 \pi}\left\{\int _ { - \infty } ^ { \infty } \frac { 1 } { R ^ { 2 } ( \omega ) + l ^ { 2 } ( \omega ) } \left[\alpha_{1}\left(X_{4}^{2}+Y_{4}^{2}\right)\right.\right. \\
& \left.\left.+\alpha_{2}\left(X_{5}^{2}+Y_{5}^{2}\right)+\alpha_{3}\left(X_{6}^{2}+Y_{6}^{2}\right)\right] d \omega\right\}, \\
\sigma_{w}^{2}= & \frac{1}{2 \pi}\left\{\int _ { - \infty } ^ { \infty } \frac { 1 } { R ^ { 2 } ( \omega ) + l ^ { 2 } ( \omega ) } \left[\alpha_{1}\left(X_{7}^{2}+Y_{7}^{2}\right)\right.\right. \\
+ & \left.\left.\alpha_{2}\left(X_{8}^{2}+Y_{8}^{2}\right)+\alpha_{3}\left(X_{9}^{2}+Y_{9}^{2}\right)\right] d \omega\right\} .
\end{aligned}
$$

If we are interested in the dynamics of system (17) with either $\alpha_{1}=0$ or $\alpha_{2}=0$, then the population variances are:

If $\alpha_{1}=0$, then

$$
\begin{aligned}
& \sigma_{u}^{2}=\frac{\alpha_{2}\left(X_{2}^{2}+Y_{2}^{2}\right)}{2 \pi} \int_{-\infty}^{\infty} \frac{1}{R^{2}(\omega)+l^{2}(\omega)} d \omega, \\
& \sigma_{v}^{2}=\frac{\alpha_{2}\left(X_{4}^{2}+Y_{4}^{2}\right)}{2 \pi} \int_{-\infty}^{\infty} \frac{1}{R^{2}(\omega)+l^{2}(\omega)} d \omega .
\end{aligned}
$$

If $\alpha_{2}=0$, then

$$
\begin{aligned}
& \sigma_{u}^{2}=\operatorname{frac}_{1}\left(X_{1}^{2}+Y_{1}^{2}\right) 2 \pi \int_{-\infty}^{\infty} \frac{1}{R^{2}(\omega)+l^{2}(\omega)} d \omega, \\
& \sigma_{v}^{2}=\frac{\alpha_{1}\left(X_{3}^{2}+Y_{3}^{2}\right)}{2 \pi} \int_{-\infty}^{\infty} \frac{1}{R^{2}(\omega)+l^{2}(\omega)} d \omega .
\end{aligned}
$$

Finally we conclude that Fourier transform method which has been used to study the effects of stochasticity on the positive equilibrium of our model leading to chaos in a realistic ecological situation.

\section{Stochastic scenario of delayed system}

The prey-predator system (1) with delay in a random environment will be considered as:

$$
\begin{aligned}
& \frac{d P}{d t}=m_{1} P\left(1-\frac{P}{K_{P}}\right)-g_{s} \frac{P Z}{P+K_{Z}}+\alpha_{1} \eta_{1}(t), \\
& \frac{d Z}{d t}=a g_{s} \frac{P(t-\tau) Z}{P(t-\tau)+K_{Z}}-m_{2} Z-g_{w} \frac{Z F}{Z+K_{F}}+\alpha_{2} \eta_{2}(t), \\
& \frac{d F}{d t}=g_{w} \frac{Z(t-\tau) F}{Z(t-\tau)+K_{F}}-m_{3} F+\alpha_{3} \eta_{3}(t) .
\end{aligned}
$$

Again using the transformations: $P^{\prime}=\ln P, Z^{\prime}=\ln Z$, $F^{\prime}=\ln F ; \quad P=u+P^{*}, Z=v+Z^{*}, F=w+F^{*} \quad$ and assuming the delay to be very small, the system (38) (to a first approximation) can be written as

$$
\begin{aligned}
& \frac{d u}{d t}=a_{11} u(t)+a_{12} v(t)+\alpha_{1} \eta_{1}(t), \\
& \frac{d v}{d t}=a_{22} v(t)+a_{23} w(t)+b_{21} u(t-\tau)+\alpha_{2} \eta_{2}(t), \\
& \frac{d w}{d t}=b_{32} v(t-\tau)+\alpha_{3} \eta_{3}(t)
\end{aligned}
$$

where, $\quad a_{11}=-m_{1} \frac{P^{*}}{K_{P}}+g_{s} \frac{P^{*} Z^{*}}{\left(P^{*}+K_{Z}\right)^{2}}, \quad a_{12}=g_{s} \frac{P^{*}}{P^{*}+K_{Z}}$, $a_{22}=g_{w} \frac{Z^{*} F^{*}}{\left(Z^{*}+K_{F}\right)^{2}}, a_{23}=g_{w} \frac{Z^{*}}{Z^{*}+K_{F}}, b_{21}=a g_{s} \frac{K_{Z} Z^{*}}{\left(Z^{*}+K_{Z}\right)^{2}}, b_{32}=$ $g_{w} \frac{F^{*} K_{F}}{\left(Z^{*}+K_{F}\right)^{2}}$.

Taking Fourier transform of both sides of each of the equation in (39), we obtain

$$
\begin{aligned}
& \alpha_{1} \tilde{\eta}_{1}(\omega)=\left(i \omega+a_{11}\right) \tilde{u}(\omega)+a_{12} \tilde{v}(\omega), \\
& \alpha_{2} \tilde{\eta}_{2}(\omega)=b_{21} \tilde{u}(\omega) e^{-i \omega \tau}+\left(i \omega+a_{22}\right) \tilde{v}+a_{23} \tilde{w}(\omega), \\
& \alpha_{3} \tilde{\eta}_{3}(\omega)=b_{32} \tilde{v}(\omega) e^{-i \omega \tau}+i \omega \tilde{w},
\end{aligned}
$$

where $\bar{n}(\omega)=\int_{-\infty}^{+\infty} n(t) e^{-i \omega t} d t$.

The system of Eq. (40) can be written in matrix form as

$$
A X=B \text {, }
$$

where

$$
\begin{aligned}
A & =\left(\begin{array}{ccc}
i \omega+a_{11} & a_{12} & 0 \\
b_{21} e^{-i \omega \tau} & i \omega+a_{22} & a_{23} \\
0 & b_{32} e^{-i \omega \tau} & i \omega
\end{array}\right), \\
X & =\left(\begin{array}{c}
\bar{u}(\omega) \\
\bar{v}(\omega) \\
\bar{w}(\omega)
\end{array}\right) \quad \text { and } \quad B=\left(\begin{array}{c}
\alpha_{1} \tilde{\eta}_{1} \\
\alpha_{2} \tilde{\eta}_{2} \\
\alpha_{3} \tilde{\eta}_{3}
\end{array}\right) .
\end{aligned}
$$

Now assuming $A^{-1}$ exists, we have $A^{-1}=\left(A_{i j}\right)_{3 \times 3}$, then the solution of (41) can be written as

$$
\begin{gathered}
\bar{u}(s)=\sum_{j=1}^{3} A_{1 j} \alpha_{j} \tilde{\eta}_{j}, \quad \bar{v}(s)=\sum_{j=1}^{3} A_{2 j} \alpha_{j} \tilde{\eta}_{j} \\
\bar{w}(s)=\sum_{j=1}^{3} A_{3 j} \alpha_{j} \tilde{\eta}_{j} .
\end{gathered}
$$

Now following Samanta (1996) and Nisbet and Gurney (1982) and using Eq. (26), the spectral density of $u$ is given by

$$
\begin{aligned}
S_{u}(\omega) & =\lim _{T \rightarrow \infty} \frac{1}{T} \int_{-T / 2}^{T / 2} \int_{-T / 2}^{T / 2}\left\langle u(t) u\left(t^{\prime}\right)\right\rangle \exp \left\{i \omega\left(t^{\prime}-t\right)\right\} d t d t^{\prime} \\
& =\sum_{j=1}^{3}\left|A_{1 j}\right|^{2} S_{\eta_{j}}(\omega) .
\end{aligned}
$$

Similarly the spectral density of $v$ and $w$ given by 
Fig. 1 Time series (a) and phase space (b) diagram of system (1), figures show that system is asymptotically stable. Other parameters are as in the text

Fig. 2 Time series solutions of system (7), a, b show that system (7) is stable and unstable for $\tau=0.3<\tau_{0}=$ 0.845 and $\tau=1.2>\tau_{0}=0.845$ respectively. Other parameters are as in the text

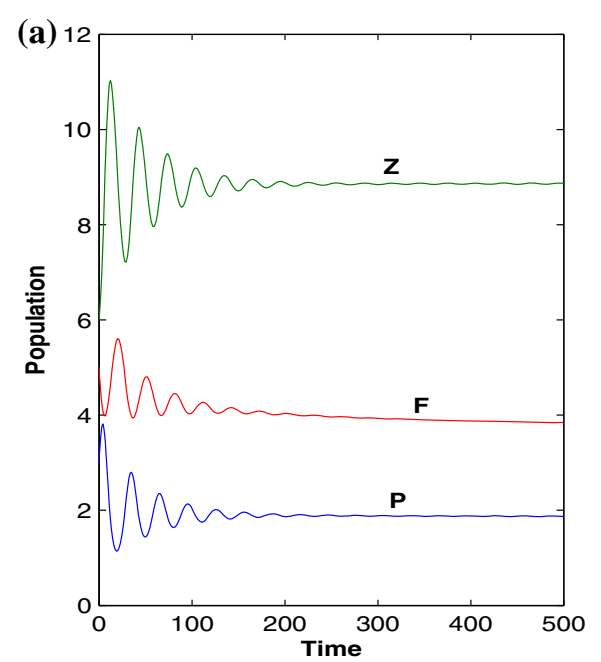

(b)
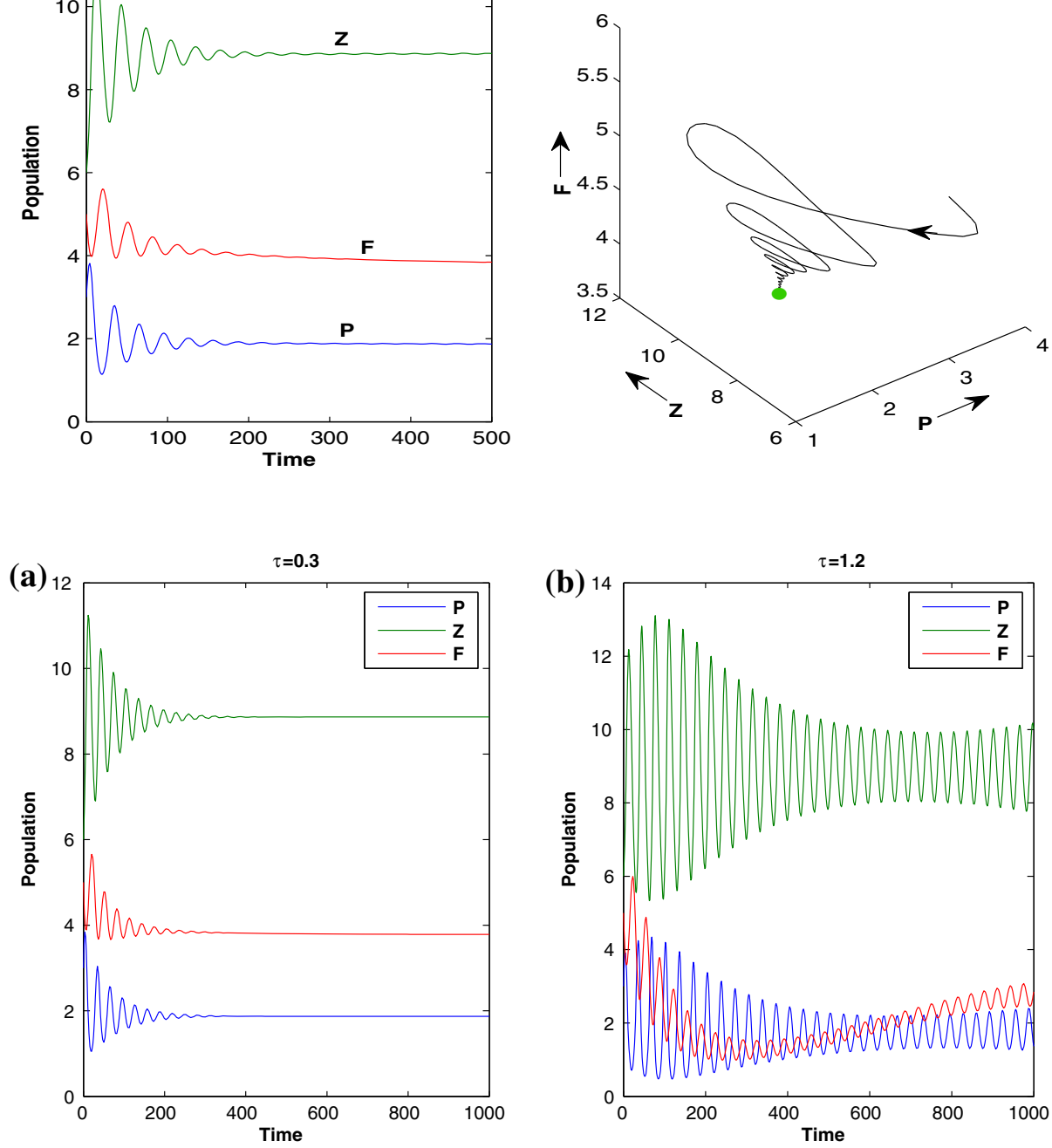

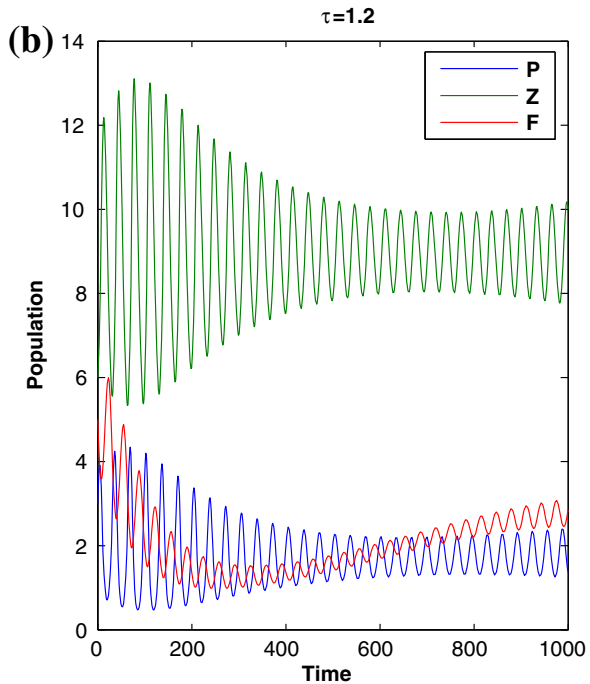

$S_{v}=\sum_{j=1}^{3}\left|A_{2 j}\right|^{2} S_{\eta_{j}}(\omega) \quad S_{w}=\sum_{j=1}^{3}\left|A_{3 j}\right|^{2} S_{\eta_{j}}(\omega)$.

Therefore the fluctuation intensity (variance) of $u$ is given by

$$
\begin{aligned}
\sigma_{u}^{2} & =\frac{1}{2 \pi} \int_{-\infty}^{+\infty} S_{u}(\omega) d \omega=\frac{1}{2 \pi} \int_{-\infty}^{+\infty} \sum_{j=1}^{3}\left|A_{1 j}\right|^{2} S_{\eta_{j}}(\omega) d \omega \\
& =\frac{1}{2 \pi} \int_{-\infty}^{+\infty} \sum_{j=1}^{3}\left|A_{1 j}\right|^{2} d \omega
\end{aligned}
$$

since $S_{\eta_{j}}(\omega)=1$.

Similarly the fluctuation intensity of $v$ and $w$ are given by

$\sigma_{v}^{2}=\frac{1}{2 \pi} \int_{-\infty}^{+\infty} \sum_{j=1}^{3}\left|A_{2 j}\right|^{2} d \omega \quad \sigma_{w}^{2}=\frac{1}{2 \pi} \int_{-\infty}^{+\infty} \sum_{j=1}^{3}\left|A_{3 j}\right|^{2} d \omega$.

\section{Numerical results}

In this study, we choose a parameter set as $m_{1}=0.6, m_{2}=$ $0.0698, m_{3}=0.324, g_{s}=0.75, g_{w}=0.6894, s_{e}=12.30$, $s_{r}=8.25, k_{p}=12, k_{z}=38, k_{f}=10, a=0.8$, initial data as $(3,6,5)$. By this set of parameters we get equilibrium abundance as $P^{*}=1.883, Z^{*}=8.892, F^{*}=3.798$. By this set of parameters coexistence equilibrium point $E^{*}\left(P^{*}, Z^{*}, F^{*}\right)$ of non-delayed system (1) is asymptotically stable. We can show this dynamical aspect by time series in Fig. 1a and phase space diagram in Fig. 1b. All the solutions with initial data converges to coexistence equilibrium point $E^{*}\left(P^{*}, Z^{*}, F^{*}\right)$ which is denoted by green bullet in Fig. 1b. Now we move on to the delayed system (2) with the same parameter set. Here we can calculate $\omega_{0}=0.327$ and critical value of delay parameter $\tau_{0}=0.845$. Now we choose $\tau=0.3$ and $\tau=1.2$, here we observe by time series solution that system (2) is stable for $\tau=0.3$ (Fig. 2a) and 


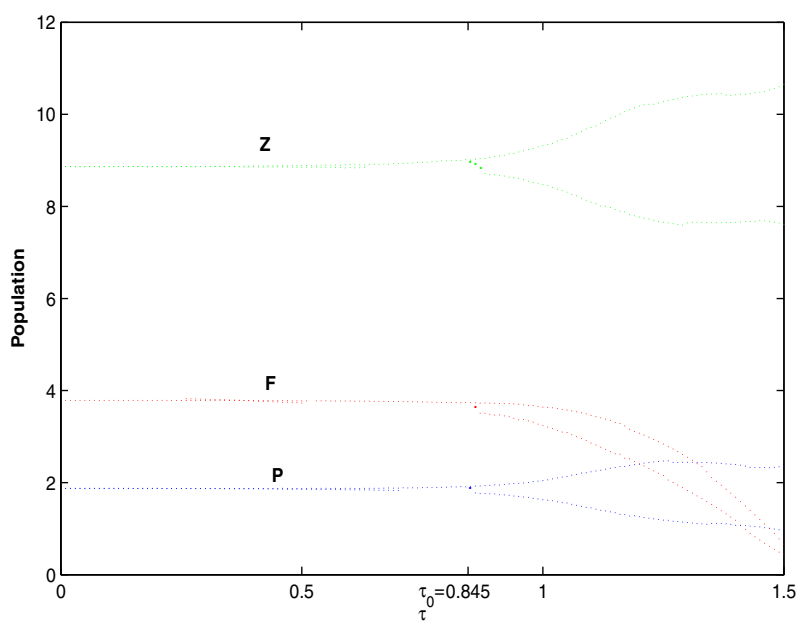

Fig. 3 Bifurcation diagram of system (7) with respect to $\tau$, system (7) is stable and unstable when $\tau<\tau_{0}=0.845$ and $\tau>\tau_{0}=0.845$ respectively and system experiences Hopf bifurcation when $\tau=\tau_{0}=0.845$. Other parameters are as in the text

unstable for $\tau=1.2$ (Fig. 2b). Broadly we show the system (2) with respect to long range of $\tau$ by bifurcation diagram in Fig. 3, this figure indicates that system (2) is stable for $\tau<\tau_{0}=0.845$ and unstable for $\tau>\tau_{0}=0.845$ and system experiences Hopf bifurcation at $\tau=\tau_{0}=0.845$.

In the numerical experiments of the stochastic system, we consider the set of parameter values previously with $\alpha_{1}=0.3, \alpha_{2}=0.2, \alpha_{3}=0.27$ and approximate the solutions of the system (38) by Euler-Maruyama method.
Delay-stochastic system (38) is stochastically stable for $\tau_{1}=0.3$ (see time series in Fig. $4 \mathrm{a}$ and phase portrait in Fig. 4b) and stochastically unstable for $\tau_{2}=1.2$ (see time series in Fig. 5a and phase portrait in Fig. 5b).

\section{Discussion}

In this present work, we consider a $P Z F$-model with Holling type II functional response where photosynthesis rate of phytoplankton, salinity induced grazing rate of zooplankton and gestation delay of zooplankton and fish population are considered. In the later part of the study, we study the impact of stochastic perturbation of the environment on the system by using Gaussian white noise. Delay induced deterministic system system shows that there is a critical value of delay parameter bellow which system is stable and above which system is unstable and when the delay parameter crosses the critical value then system experiences Hopf-bifurcation.

The main purpose of this work is to observe the effect of delay along with photosynthesis rate of phytoplankton, salinity induced grazing rate of zooplankton under fluctuating environment. Results show that these biological parameters play a significant role to change the stochastic stability of the system. To study the effect of environmental fluctuation on the non-delayed $P Z F$ system, we have superimposed Gaussian white noises and then studied non-equilibrium fluctuation and stability of the resulting
Fig. 4 Plots $\mathbf{a}$ and $\mathbf{b}$ depict, respectively, the time evolution and corresponding phase trajectory of the delaystochastic model system (38). For $\alpha_{1}=0.3, \alpha_{2}=0.2$, $\alpha_{3}=0.27$, system is stochastically stable for $\tau=0.3$. Other parameters are as in the text

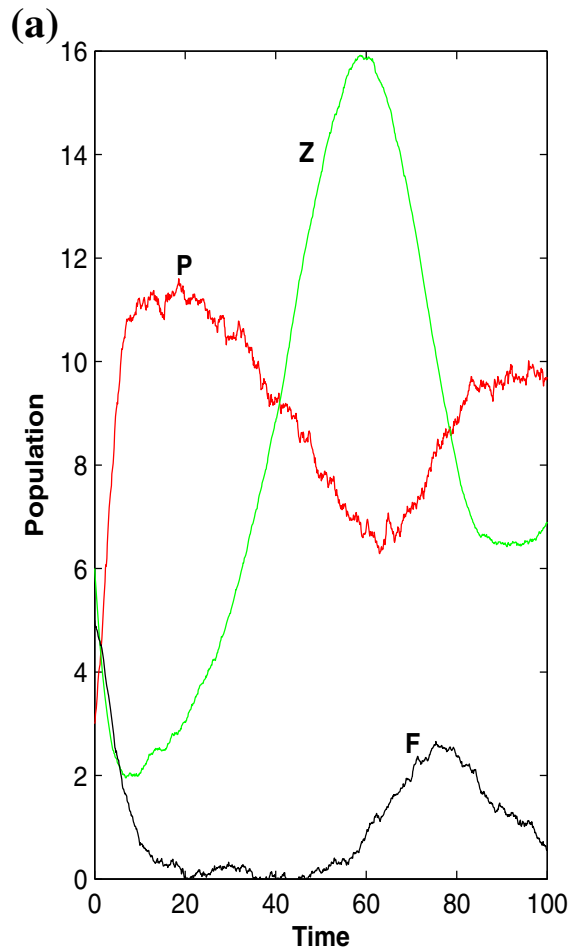

(b)

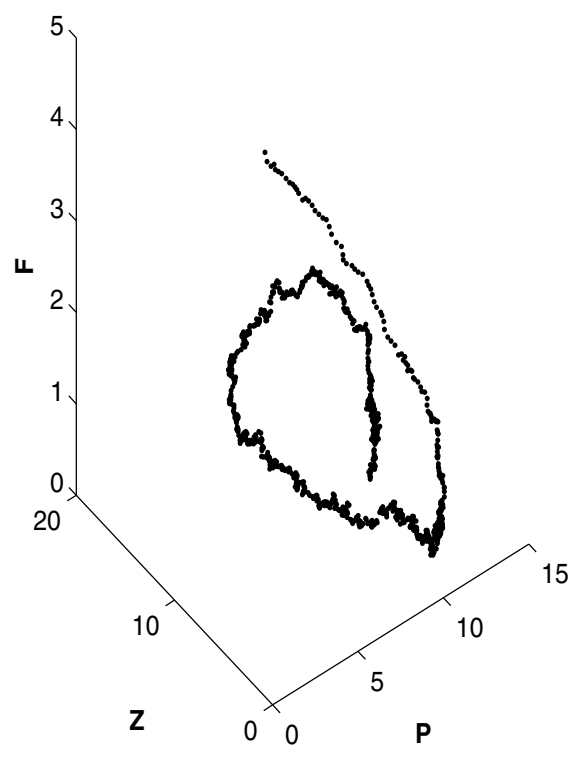


Fig. 5 Plots a and $\mathbf{b}$ depict, respectively, the time evolution and corresponding phase trajectory of the delaystochastic model system (38). For $\alpha_{1}=0.3, \alpha_{2}=0.2$, $\alpha_{3}=0.27$, system is stochastically unstable for $\tau=1.2$. Other parameters are as in the text

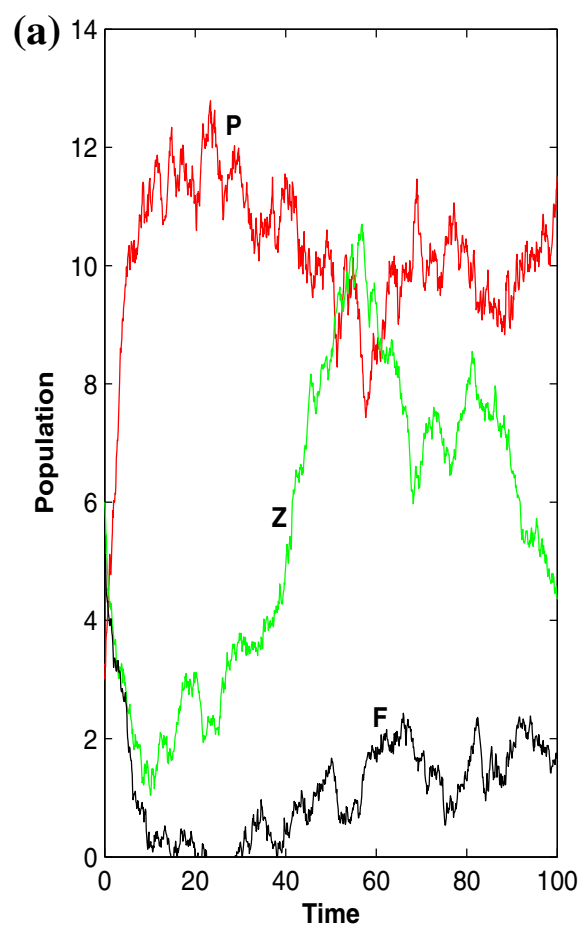

stochastic model by using Fourier transform technique. Also the deterministic system and the noise-induced stochastic system may be have a like with respect to stability.

\section{References}

Berryman AA (1992) The origins and evolution of predatorprey theory. Ecology 73:1530-1535

Ding Y, Jiang W (2011) Double Hopf bifurcation and chaos in Liusystem with delaed feedback. J Appl Anal Comput 1(3):325-349

Ding Y, Jiang W, Yu P (2013) Double Hopf bifurcation in acontainer crane model with delayed position feedback. Appl Math Comput 219:9270-9281

Iwasa Y, Hakoyama H, Nakamaru M, Nakanishi J (2000) Estimate of population extinction risk and its application to ecological risk management. Popul Ecol 42:73-80

Jana D (2014) Stabilizing effect of prey refuge and predator's interference on the dynamics of prey with delayed growth and generalist predator with delayed gestation. Int J Ecol 2014:12. Article ID 429086. doi:10.1155/2014/429086

Jana D, Bairagi N (2013) complexity, stochasticity and the stability of predator-prey interactions. J Control Eng Technol 3(2):76-83

Jana D, Agrawal R, Upadhyay RK (2014) Top-predator interference and gestation delay as determinants of the dynamics of a realistic model food chain. Chaos Solitons Fractals 69:50-63

Jana D, Agrawal R, Upadhyay RK (2015) Dynamics of generalist predator in a stochastic environment: effect of delayed growth and prey refuge. Appl Math Comput 268:1072-1094

Ketchum BH (1951) The exchange of fresh and salt water in tidal estuaries. J Mar Res 10:1838

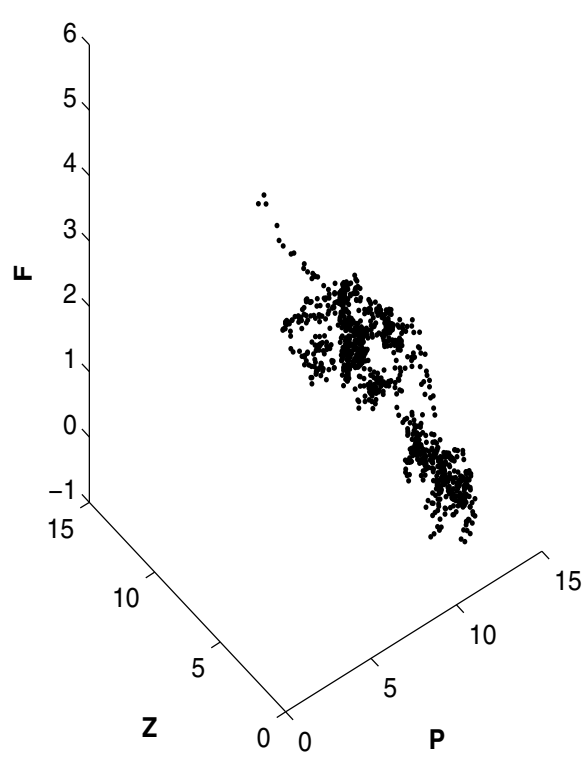

(b)

Lande R (1993) Risks of population extinction from demographic and environmental stochasticity and random catastrophes. Am Nat 142:911-927

Lü J, Chen G, Cheng D, Celikovsky S (2002) Bridge the gap between the Lorenz system and the Chen system. Int J Bifurc Chaos 12:2126-2917

Ma S, Lu Q, Feng Z (2008) Double Hopf bifurcation for van der PolDuffing oscillator with parametric delay feedback control. J Math Anal Appl 338:993-1007

Mackey M, Glass L (1977) Oscillations and chaos in physiological control systems. Science 197:287-289

Mandal S, Ray S, Ghose PB (2012) Modelling nutrient (dissolved inorganic nitrogen) and plankton dynamics at sagarisland of Hooghly-Matla estuarine system, West Bengal, India. J Nat Resour Model 25:629-652

Matouk AE (2008) Dynamical analysis, feedback control synchronization of Liu dynamical system. Nonlinear Anal 69:3132-3213

May RM (1973) Stability in randomly fluctuating deterministic environments. Am Nat 107:621-650

Nisbet RM, Gurney WSC (1982) Modelling fluctuating populations. Wiley Interscience, NewYork

Ripa J, Lundberg P, Kaitala V (1998) A general theory of environmental noise in ecological food webs. Am Nat 107:256-263

Ruan S (2009) On nonlinear dynamics of predator-prey models with disc rete delay. Math Model Nat Phenom 4(2):140-188

Samanta GP (1996) The effect of random fluctuating environment on interacting species with time delay. Int J Math Educ Sci Technol 27:13-21

Samanta GP, Maiti A (2003) Stochastic Gomatam model of interacting species: non-equilibrium fluuctuation and stability. Syst Anal Model Simul 43:683-692

Schwartz IB, Billings L, Carr TW, Dykman MI (2015) Noise-induced switching and extinction in systems with delay. Phys Rev E 91:121-139 
Song Z, Xu Z (2013) Stability switches and double Hopf bifurcation in a two-neural network system with multiple delays. Cogn Neurodyn 7:505-521

Turelli M (1986) Stochastic community theory: a partially guided tour. In: Hallman TG, Levin S (eds) Mathematical ecology. Springer, Berlin

Upadhyay RK, Mukhopadhyay A, Iyengar SRK (2007) Influence of environmental noise on the dynamics of a realistic ecological model. Fluct Noise Lett 7(01):61-77
Valsakumar MC, Murthy KPN, Ananthakrishna G (1983) On the linearization of non-linear Langevin type stochastic differential equations. J Stat Phys 30:617-631

Wang Y, Wang H, Jiang W (2014) Hopf-transcritical bifurcation in toxic phytoplankton-zooplankton model with delay. J Math Anal Appl 415:574-594 